\title{
Role of hospital supportive services in COVID-19
}

\author{
Jitender Sodhi ${ }^{1}$, Sidhartha Satpathy ${ }^{1}$, Pankaj Arora ${ }^{2}$ \\ ${ }^{1}$ Department of Hospital Administration, All India Institute of Medical Sciences, New Delhi, India \\ ${ }^{2}$ Department of Hospital Administration, \\ Postgraduate Institute of Medical Education and Research, Chandigarh, India
}

DOI: 10.3396/ijic.v16i3.022.20

\begin{abstract}
While clinical services of a hospital have to respond to the COVID-19 pandemic and flow of COVID-positive patients into makeshift wards and critical care units of the hospital, supportive services and their staff have to gear up to complement the life-saving efforts of front-line providers. The spectrum of supportive services to a hospital encompasses linen and laundry, dietary, Central Sterile Supply Department (CSSD), transport, hospital stores, mortuary and engineering services.
\end{abstract}

Each of these services has a significant role to help abort the 'chain of transmission' of COVID-19 infection across various patient care areas in the hospital, while providing them supportive services. The linen and laundry service with its robust infection control process in situ, brings back into the circulation of clinical care units, clean and infection free bed and body linen which is used for COVID-19 patients. Clean and infection free linen inspires confidence amongst the patients and enhances their satisfaction level. ${ }^{1}$ The dietetics department, which serves hygienic and nutritious diets to patients in isolation rooms or wards and the healthcare personnel on duty at the COVID-19 wards, is playing an indispensable role in a pandemic situation, as the healthcare personnel cannot go out from the clinical units and have to be served meals there. The importance of nutrition in acute and chronic diseases, particularly in ailments in which an etiologic treatment has not yet been discovered and validated is well in place. ${ }^{2}$ The safe transport of patients, either suspected or confirmed cases, from emergency departments to the wards/isolation rooms, intensive care units and to radiology suites requires a defined route, a designated elevator, and dedicated patient transport trolley. Likewise, inter-hospital transfer of patients may be required in certain cases, where breaches in infection control protocol can potentially lead to massive spread of infection and can defeat all the efforts undertaken to break the chain of transmission. $^{3}$ Therefore, the hospital transport services contribute to a great extent in containment of infection through its safe transfer protocols. Additionally, with the public transportation services coming to a halt due to lockdown, hospital transport has to pitch in for bringing healthcare workers to the hospital as well as bringing in materials from within the city limits and outside. Sourcing this material brings to light the difficulties faced by procurement managers in such disasters. Hospital stores may need to procure consumables in large quantities in unusual conditions, which normally were brought in minimal quantities. Some of these may be the most critical items e.g. filtering face piece respirators or 
N95 respirators and personal protective equipment (PPE) kits. The challenge in terms of inadequate availability of PPE in hospital settings throughout the world during this pandemic highlights the role of CSSD. A method of decontamination and reuse of filtering face respirators that has been suggested by Centers for Disease Control and Prevention (CDC) to overcome the shortage of these respirators is their extended use or reuse. ${ }^{4}$ CSSD has to use its expertise in sterilization techniques to make these filtering face respirators, N95 respirators available to the users after re-processing for reuse. In resource limiting conditions, reuse of other protective gear, like face shields, protective gear for eyes and footwear, can help in saving resources as well as reducing burden to the environment in the form of biomedical waste. The role of CSSD is vital during this pandemic as it is best equipped in terms of equipment as well as technical expertise to handle this task. Transfer of dead bodies of COVID-19 from wards/isolation rooms, their safe preservation and final disposal (cremation or burial) outlines the importance of a well-equipped morgue with dedicated cold cabinets and sufficient PPE in place. Most of our hospitals are not geared up physically to face such an epidemic in terms of isolation cubicles, negative pressure rooms, air conditioning, operating theatres and ICUs. Major and minor modifications need to be carried out in functioning hospitals, sometimes overnight, to make the areas suitable for patient intake. Most tertiary care hospitals have central air-conditioning with lesser air changes and minimal fresh air, which has the potential to spread SARS-CoV-2 virus. A recent report from Wuhan, China, where this pandemic originated, has shown that central air-conditioning in a restaurant spread the virus from one suspect case to many others who were present at the same time. ${ }^{5}$ Making appropriate changes like the addition of ultra violet light (Far-UVC) in the air handling units have shown promising results at Columbia University. ${ }^{6}$

In essence, it can be stated that supportive services in a hospital constitute important cogs in the wheel of clinical care given to patients in any hospital setting, and have a crucial role in mitigation of infection and delivery of safe care.

\section{Reference:}

1. Singh D, Qadri G, Kotwal M, Syed A, Jan F. Quality control in linen and laundry service at a tertiary care teaching hospital in India. Int J Health Sci (Qassim) 2009; 3(1): 33-44.

2. Laviano $A$, Koverech $A$, Zanetti M. Nutrition support in the time of SARS-CoV-2 (COVID-19). Nutrition 2020; 74: 110834. https://doi.org/10.1016/j.nut.2020.110834

3. Liew MF, Siow WT, Yau YW, See KC. Safe patient transport for COVID-19. Critical Care 2020; 24(1): 94. https://doi. org/10.1186/s13054-020-2828-4

4. Decontamination and Reuse of Filtering Facepiece Respirators. Updated April 30, 2020 . https://www.cdc.gov/ coronavirus/2019-ncov/hcp/ppe-strategy/decontaminationreuse-respirators.html. Accessed 17 June 2020.

5. Lu J, Gu J, Li K, et al. COVID-19 Outbreak Associated with Air Conditioning in Restaurant, Guangzhou, China, 2020. Emerg Infect Dis 2020; 26(7). http://wwwnc.cdc.gov/eid/ article/26/7/20-0764_article.htm. Accessed 17 June 2020.

6. Welch D, Buonanno M, Grilj V, et al. Far-UVC light: A new tool to control the spread of airborne-mediated microbial diseases. Sci Rep 2018; 8(1): 2752. https://doi.org/10.1038/ s41598-018-21058-w 